\title{
Estimación de la profundidad de trabajo de anzuelos de un palangre atunero prototipo de la flota de altura de Isla de Margarita (Venezuela)
}

\author{
Ernesto Trujillo ${ }^{1} \&$ Guillermo Martínez ${ }^{2}$ \\ ${ }^{1}$ Instituto de Investigaciones Científicas, Universidad de Oriente \\ Apartado Postal 147, Boca de Río, Venezuela \\ ${ }^{2}$ Escuela de Ciencias del Mar, Pontificia Universidad Católica de Valparaíso \\ Casilla 1020, Valparaíso, Chile \\ E-mail: etrujill@ci.udo.edu.ve; guillermo.martinez@ucv.cl
}

\begin{abstract}
RESUMEN. Sobre la base de dos campañas de pesca realizadas en el Mar Caribe, en mayo de 1997 y junio de 1998 , a bordo de una embarcación de altura palangrera atunera de Chacachacare, Isla de Margarita, Venezuela, se determinó la forma y tensión del palangre atunero en condiciones de trabajo y se estimó la profundidad de trabajo de los anzuelos mediante el método de simulación mecánica. El factor de escala lineal para la simulación de una sección del prototipo de seis anzuelos $\left(C_{L}\right)$ fue 150 y el factor de escala de fuerza $\left(C_{R}\right)$ fue 370,9. Las velocidades de la embarcación y las tasas de recorte de las secciones del palangre, para la experimentación fluctuaron entre 4,9 nudos $(55,3 \%)$ y 7,1 $(80,5 \%)$. Los ángulos de ataque medios en los terminales de la línea madre del prototipo fluctuaron entre $10,4^{\circ}( \pm 0,97$ $\mathrm{DE})$ y $29,75^{\circ}( \pm 1,59 \mathrm{DE})$, con una tensión promedio entre $2,42 \mathrm{kgf}( \pm 0,46 \mathrm{DE})$ y $5,35( \pm 1,10 \mathrm{DE})$. Los anzuelos $\mathrm{N}^{\circ} 1$ y No , en una gama de velocidades de calado de la línea principal, alcanzaron profundidades entre 63,9 y 70,4 m, los anzuelos $\mathrm{N}^{\circ} 2$ y $\mathrm{N}^{\circ} 5$ entre 89,8 y 103,8 $\mathrm{m}$ y los anzuelos $\mathrm{N}^{\circ} 3$ y $\mathrm{N}^{\circ} 4$ entre 103,2 y 133,1 m. Las profundidades estimadas de los anzuelos presentaron una relación inversa con las velocidades de calado del palangre $\left(\mathrm{V}_{\mathrm{L}}\right)$, las tasas de recorte (k) y los ángulos de ataque en los terminales de una sección de línea madre. Las tensiones horizontales estimadas presentaron una relación directa con $\mathrm{V}_{\mathrm{L}} \mathrm{y} \mathrm{k}$.
\end{abstract}

Palabras claves: palangre atunero, profundidad de anzuelos, simulación mecánica, Venezuela.

\section{Estimation of the operational depth of tuna longline hooks of the Isla Margarita (Venezuela) fishing fleet}

\begin{abstract}
Based on two fishing trips carried out in the Caribbean Sea in May 1997 and in June 1998, on board of deep sea tuna longline fishing boat, from Chacachacare, Margarita Island, Venezuela, the shape and tension of the longline were determined under working conditions, and the operational depth of hooks were estimated by mechanical simulation method. Lineal scale factor for simulation of a prototype section of six hooks $\left(\mathrm{C}_{\mathrm{L}}\right)$ was 150 and force scale factor $\left(\mathrm{C}_{\mathrm{R}}\right)$ was 370.9. Vessel speed during the operations and sections sagging rate, for trial on test panel, ranged from 4.9 knots $(55.3 \%)$ to $7.1(80.5 \%)$. The angles of incidence at the ends of the main line of prototype ranged between a mean value of $10.4^{\circ}( \pm 0.97 \mathrm{SD})$ and $29.75^{\circ}( \pm 1.59 \mathrm{SD})$, with a mean value of tension between $2.42 \mathrm{kgf}( \pm .0 .46 \mathrm{SD})$ and $5.35( \pm 1.10 \mathrm{SD})$. The hooks $\mathrm{N}^{\circ} 1$ and $\mathrm{N}^{\circ} 6$, in a range of vessel speeds, reached depths between 63.9 and $70.4 \mathrm{~m}$, the $\mathrm{N}^{\circ} 2$ and $\mathrm{N}^{\circ} 5$ reached depths between 89.8 and $103.8 \mathrm{~m}$, and the hooks $\mathrm{N}^{\circ} 3$ and $\mathrm{N}^{\circ} 4$ reached depths between 103.2 and $133.1 \mathrm{~m}$. The estimated hook depths showed an inverse relation with the speed at which the main line is set $\left(\mathrm{V}_{\mathrm{L}}\right)$, the sagging rate $(\mathrm{k})$ and the angles of incidence at the end sections of main line (joint between the buoy line and two longline sections). The estimation of horizontal tensions shows a direct relationship with $\mathrm{k}$ and $\mathrm{V}_{\mathrm{L}}$.
\end{abstract}

Key words: tuna longline, operational depth hook, mechanical simulation, Venezuela. 


\section{INTRODUCCIÓN}

En la operación de pesca con palangre, un aspecto fundamental de la táctica de pesca, es disponer los anzuelos en el estrato de la columna de agua en el cual se encuentra la especie objetivo y asegurar con ello una mayor efectividad de captura y el éxito del lance (Fridman, 1986). En efecto, considerando las características del palangre, las condiciones ambientales y el comportamiento de la especie objetivo, se debe estimar el recorte del palangre durante el calado y, así, determinar tanto la configuración como el seno que adoptará la línea madre, producto de la flecha que se forma al interactuar sobre ella distintas componentes de fuerza (Martínez et al., 1993). A mayor recorte, mayor será el seno de la línea madre y, por ende, mayor será la profundidad de calado de los anzuelos; en cambio, a menor recorte la profundidad de calado de los anzuelos será menor.

En la práctica, es posible regular el recorte mediante la velocidad de calado del palangre, es decir, a mayor velocidad se obtiene un menor recorte, quedando la línea madre más tensa; mientras que a menor velocidad de calado el recorte será mayor, produciéndose un mayor seno en la línea madre. Sin embargo, generalmente los patrones de los barcos pesqueros calan el aparejo sin considerar este efecto del recorte, estableciendo sus tácticas de pesca sobre la base de prueba y error.

Si bien es cierto que en la actualidad existe la tecnología para medir la profundidad de trabajo del palangre, mediante sensores remotos dispuestos en los anzuelos (Nishi, 1990; Mizuno et al., 1997; Okazaki et al., 1997; Matsumoto et al., 1999; Yokawa \& Takuchi, 2000; entre otros), su alto costo de inversión es una seria limitante para masificar su uso en forma operacional en una pesquería de pequeña escala.

Un método alternativo al uso de sensores, es la simulación mecánica de un modelo del palangre prototipo utilizado en la pesquería de atunes (Nemoto, 1975; Suzuki et al., 1977; Yuwaki et al., 1985; Eslava \& Gaertner, 1990; Nakano et al., 1997; Santana-Hernández et al., 1998; Bigelow et al., 2000; entre otros). El método de imitación mecánica fue desarrollado por Baranov (1939) para el diseño de artes de pesca industriales, cuyo enfoque estuvo dirigido a los sistemas de hilo flexible. En tal sentido, el fundamento de la modelación mecánica consiste en la preparación y experimentación de un modelo análogo del esquema de cálculo para un arte de pesca o de sus partes, donde las líneas geométricas en el esquema se reemplazan por hilos flexibles, a los cuales se aplican fuerzas externas en las respectivas secciones (Martínez \& Melo, 1990). De acuerdo a la acción de ellas, se determina la forma del arte de pesca y la magnitud de la reacción en los puntos de fijación.

En vista de lo expuesto, y por tratarse de una pesquería de pequeña escala, como es la realizada por la flota palangrera atunera de Chacachacare, Isla de Margarita, Venezuela, es necesario dotar a los patrones de pesca de un instrumento decisional, que les permita establecer la velocidad de calado del palangre, necesaria para que los anzuelos queden trabajando a la profundidad deseada. Por esta razón, el objetivo principal de este trabajo es determinar la forma y tensión del palangre en condiciones de trabajo y estimar la profundidad teórica de operación de los anzuelos, utilizando el método de simulación mecánica o análoga.

\section{MATERIALES Y MÉTODOS}

\section{Simulación mecánica de una sección de línea madre}

Para realizar la simulación mecánica, se utilizó una cadena flexible como modelo del perfil de una sección de línea madre, con su correspondiente escala lineal $\left(C_{L}\right)$. Las fuerzas externas se imitaron en forma de pesos, sustentadas en su respectiva escala de fuerzas $\left(\mathrm{C}_{\mathrm{R}}\right)$. Mediante el equilibrio del sistema con ayuda de pesos, la cadena flexible adoptó la forma buscada y el sistema de los pesos en equilibrio demostró, sobre las fuerzas externas, la tensión en las uniones y sobre los demás factores de fuerza, lo cual se basó en dos principios (Voinikanis-Mirskii, 1979):

- En los sistemas estáticos de articulaciones definibles, las fuerzas en los sistemas no dependen de las medidas de éstos.

- Ante el cambio de todas las cargas aplicadas, en una relación determinada, las fuerzas en los elementos del sistema cambian en la misma proporción.

Por lo tanto, las constantes geométricas y de fuerzas se consideraron independientes entre sí, por lo que el modelo fue ejecutado de una forma, y las fuerzas fueron ejecutadas, independientemente, en otra escala. En tal sentido, las escalas se determinaron de acuerdo a la comodidad en la elaboración del modelo análogo y la realización del experimento, 
según lo recomendado por Baranov (1969) y Voinikanis-Mirskii (1979).

\section{Forma del palangre prototipo y posición de los anzuelos}

La forma de una sección de palangre fue analizada como una catenaria, lo cual permitió determinar la profundidad $\mathrm{H}_{\mathrm{A}}$ de los anzuelos centrales en la columna de agua (Fig. 1), mediante la ecuación (1):

$$
\mathrm{H}_{\mathrm{A}}=\mathrm{H}_{\mathrm{B}}+\mathrm{f}+\mathrm{L}_{\mathrm{R}}
$$

donde $\mathrm{H}_{\mathrm{A}}$ es la profundidad del anzuelo, $\mathrm{H}_{\mathrm{B}}$ es el cabo de boya, f corresponde al seno de la línea madre y $L_{R}$ es la longitud del reinal.

Durante la calada de la sección de un palangre (Fig. 1), con una longitud determinada (S), la profundidad $\left(\mathrm{H}_{\mathrm{A}}\right)$ se puede regular, hasta cierto límite, dependiendo de la medida establecida en la distancia horizontal entre las balizas A y B (L). La cuerda L va a depender de la velocidad de la embarcación cuando se lanza o cala el palangre $\left(\mathrm{V}_{\mathrm{L}}\right)$ y el tiempo

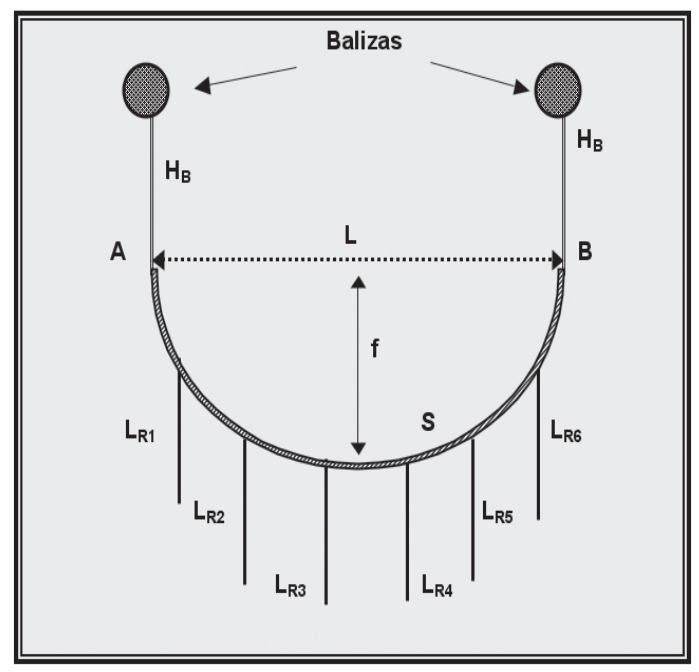

Figura 1. Configuración de una sección de línea madre con 6 anzuelos. $H_{B}$ : cabo de boya, $L$ : distancia horizontal entre boyas, S: longitud de una sección de línea madre con 6 anzuelos, f: seno de la línea madre, $\mathrm{L}_{\mathrm{Ri}}$ : longitud del reinal i, A y B: apoyos del prototipo.

Figure 1. Configuration of a section of main line with 6 hooks. $\mathrm{H}_{\mathrm{B}}$ : Buoy line, L: horizontal length between buoys, $\mathrm{S}$ : length of a section of main line with 6 hooks, $f$ : sag of the main line, $\mathrm{L}_{\mathrm{Ri}}$ : length of the branch line $\mathrm{i}, \mathrm{A}$ and $\mathrm{B}$ : prototype support. transcurrido en el calado de una sección (t), como se puede apreciar en la ecuación (2).

$$
\mathrm{L}=\mathrm{V}_{\mathrm{L}} \cdot \mathrm{t}
$$

De acuerdo con el valor de la tasa de recorte $\mathrm{k}$, definida como L/S (cociente entre la distancia horizontal entre dos flotadores y la longitud estirada de la línea madre), se puede determinar en forma preliminar la magnitud de f en una Tabla de Elementos de la Catenaria y, a partir de este valor, se puede estimar $\mathrm{H}_{\mathrm{A}}$ mediante la ecuación (1).

\section{Criterios para la simulación}

A fin de determinar los criterios de simulación de una sección del palangre prototipo para la pesca de atún, se tomó como referencia el trabajo de Nemoto (1975) para el cálculo de la profundidad de anzuelos, de acuerdo a la tasa de recorte del palangre. Asimismo, para la simulación en el laboratorio de los componentes de semejanza de fuerza (peso) y geométrica (forma) a escala, se utilizó la recomendación dada por Baranov (1969) y Fridman (1986). De acuerdo a esto, se definió el procedimiento de entrada para la simulación de un hilo flexible, en este caso una cadena.

\section{Características de la operación de pesca}

Sobre la base de la información operacional de una embarcación prototipo de altura palangrera atunera de Chacachacare, obtenida en dos campañas de pesca realizadas en el Mar Caribe en mayo de 1997 y junio de 1998. Los siguientes parámetros se consideraron para determinar la configuración del palangre:

- Tiempo promedio de calado de una sección $=1,49 \mathrm{~min}$

- Distancia promedio de recorrido de una sección $=184,53 \mathrm{~m}$

- Velocidad promedio de calado de una sección $=5,97$ nudos

\section{Modelamiento en laboratorio}

En el Laboratorio de Tecnología Pesquera de la Escuela de Ciencias del Mar de la Pontificia Universidad Católica de Valparaíso, se simuló una sección de palangre de acuerdo al régimen de calado de la embarcación prototipo. Para tal efecto, se utilizó el procedimiento de simulación análoga adoptado por 
Martínez \& Carroza (1992), para la construcción del modelo.

\section{Materiales y equipos para la simulación}

Para el montaje y desarrollo de la simulación, se construyó un panel de prueba vertical de madera de $2,42 \times 1,50 \mathrm{~m}$ (superficie de $3,63 \mathrm{~m}^{2}$ ). En éste se incorporó un juego de poleas para el deslizamiento de los pesos, bajo distintas tasas de recorte (excursionamiento), cuyas cargas fueron medidas con ayuda de una balanza analítica de $1.200 \mathrm{~g}$ de capacidad y $0,1 \mathrm{~g}$ de precisión. Para la medición de los ángulos de ataque se utilizó un transportador de sonda.

En virtud de las características del material utilizado en la construcción del palangre prototipo de seis anzuelos, se estableció el patrón de hundimiento, sobre la base de la densidad de este material. Por lo tanto, considerando que más del $90 \%$ del material utilizado es de PVA cuya densidad es $\rho=1.300$ $\mathrm{kg} / \mathrm{m}^{3}$ (Voinikanis-Mirskii, 1979), se supuso esta propiedad física para todo el palangre, cuyo peso promedio de las secciones en el aire, fue de $14,7 \mathrm{~kg}$.

\section{Definición de la escala del modelo}

Se definió la escala geométrica o lineal y de fuerza, considerando el espacio disponible y la comodidad para la construcción del modelo y el desarrollo de la experiencia.

De acuerdo a las recomendaciones de Baranov (1969) y Fridman (1973), con relación a la calidad de hilo flexible para la simulación, se utilizó una cadena metálica, cuya longitud fue de $1,82 \mathrm{~m}$ y un peso de 39,63 g. La escala lineal se determinó de la siguiente manera:

\section{Factor de escala lineal}

Tomando como unidad prototipo una sección de palangre $\left(\mathrm{L}_{\mathrm{n}}\right)$ de seis anzuelos, igual a $273 \mathrm{~m}$ y la longitud del modelo $\left(\mathrm{L}_{\mathrm{m}}\right)$ igual a $1,82 \mathrm{~m}$, el factor de escala lineal se calculó con la ecuación (3).

$$
\begin{aligned}
& \mathrm{C}_{\mathrm{L}}=\mathrm{L}_{\mathrm{n}} / \mathrm{L}_{\mathrm{m}} \\
& \mathrm{C}_{\mathrm{L}}=273 \mathrm{~m} / 1,82 \mathrm{~m} \\
& \mathrm{C}_{\mathrm{L}}=150
\end{aligned}
$$

\section{Factor de escala de peso}

De igual forma, tomando como unidad prototipo $\left(\mathrm{F}_{\mathrm{n}}\right)$ una sección de palangre de seis anzuelos, cuyo peso promedio es de $14,7 \mathrm{~kg}$ y el peso del modelo elegido $\left(\mathrm{F}_{\mathrm{m}}\right)$ igual $39,63 \mathrm{~g}$, el factor de escala de fuerza se calculó con la ecuación (4).

$$
\begin{aligned}
& \mathrm{C}_{\mathrm{R}}=\mathrm{F}_{\mathrm{n}} / \mathrm{F}_{\mathrm{m}} \\
& \mathrm{C}_{\mathrm{R}}=14.700 \mathrm{~g} / 39,63 \mathrm{~g} \\
& \mathrm{C}_{\mathrm{R}}=370,9
\end{aligned}
$$

De acuerdo a las medidas preestablecidas, se ocupó una superficie útil en el panel de prueba de $3,18 \mathrm{~m}^{2}$ (Fig. 2).

\section{Procedimiento para realizar la experiencia}

Los terminales de la cadena se fijaron en los apoyos A y B en forma simétrica (Fig. 3). Al extremo (A) se unieron dos hilos flexibles ligeros (R390tex), donde uno de ellos se pasó a través de una polea, en cuyo extremo se unió un recipiente para determinar la componente horizontal y el otro hilo se fijó a un perno, equidistante a la polea, con la finalidad de simular el componente vertical, el cual representó una fuerza equivalente a la fuerza de empuje hacia arriba (flotación) de la baliza prototipo, que se supuso constante. Según Fridman (1986):

$$
T=\sqrt{\left(T_{H}\right)^{2}+\left(T_{V}\right)^{2}}=\sqrt{\left(W_{A H}\right)^{2}+\left(W_{A V}\right)^{2}}
$$

donde $\mathrm{T}$ es la tensión en la dirección de la tangente sobre la curva en el apoyo (A), $\mathrm{T}_{\mathrm{H}}$ es la componente de tensión horizontal, $\mathrm{T}_{\mathrm{V}}$ es la componente de tensión vertical, $\mathrm{W}_{\mathrm{AH}}$ corresponde a la tensión de prueba y $\mathrm{W}_{\mathrm{AV}}$ es la tensión del primer flotador.

Como elemento de carga se utilizó arena de duna, la cual se vertió en el recipiente para obligar al terminal de la cadena a desplazarse en sentido horizontal (hacia la izquierda) hasta coincidir con el centro de la coordenada, apoyo (A) (Fig. 3). De esta forma las fuerzas aplicadas en los extremos de la

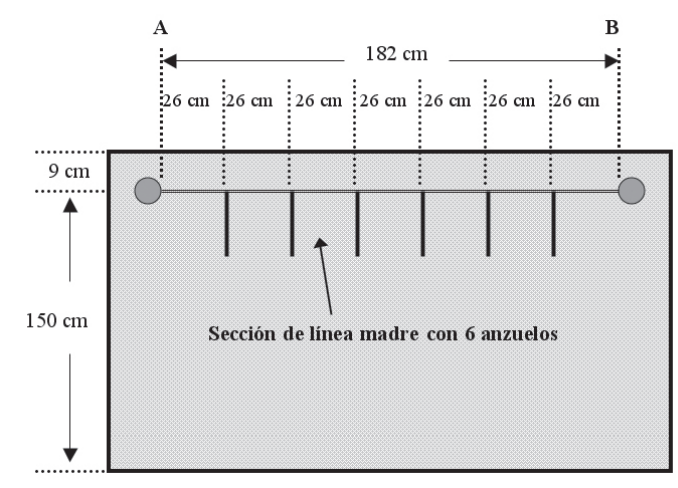

Figura 2. Panel de prueba. A y B: apoyos del prototipo. Figure 2. Test board. A and B: prototype support. 


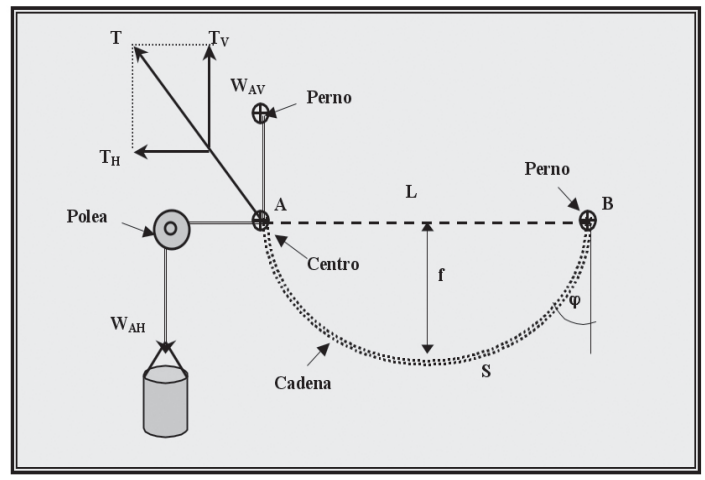

Figura 3. Modelo utilizado en la simulación mecánica (Adaptado de Baranov, 1969 y Fridman, 1986). L: distancia horizontal entre boyas, $S$ : longitud de una sección de línea madre con 6 anzuelos, f: seno de la línea madre, $\varphi$ : ángulo de la catenaria, A y B: apoyos del prototipo, T: tensión resultante, $T_{\mathrm{V}}$ : componente de tensión vertical, $\mathrm{T}_{\mathrm{H}}$ : componente de tensión horizontal, $\mathrm{W}_{\mathrm{AV}}$ : tensión de la primera boya, $\mathrm{W}_{\mathrm{AH}}$ : tensión de prueba.

Figure 3. Model used in mechanical simulation (Adapted from Baranov, 1969 and Fridman, 1986). L: horizontal length between buoys, S: length of a section of main line with 6 hooks, f: sag of the main line, $\varphi$ : catenary angle, $A$ and $B$ : prototype supports, $\mathrm{T}$ : resultant tension, $\mathrm{T}_{\mathrm{v}}$ : vertical tension component, $T_{H}$ : horizontal tension component, $W_{A V}$ : buoy tension, $\mathrm{W}_{\mathrm{AH}}$ : test tension.

cadena fueron las propias reacciones de las componentes verticales y horizontales. Para el caso del apoyo (B), cuya posición hace simétrica la catenaria, se desplazó hacia la izquierda por la cuerda (L), de acuerdo a la tasa de recorte (excursionamiento en el panel de prueba) preestablecida, siendo las fuerzas resultantes en el apoyo (B) semejantes a las del apoyo (A).

Para propósitos de la experimentación, se consideraron como velocidades de calado del palangre 7,$1 ; 6,6 ; 6,0 ; 5,8 ; 5,3$ y 4,9 nudos; las que determinaron tasas de recorte de 80,$5 ; 74,9 ; 70,0 ; 65,5 ; 60,2$ y $55,3 \%$, respectivamente.

\section{RESULTADOS}

Basados en los resultados obtenidos en las pruebas de simulación, se configuró la catenaria para seis anzuelos, en función de la velocidad de calado del palangre (Fig. 4). De acuerdo a las medidas estándares del palangre utilizado como prototipo, se determinó que los anzuelos $\mathrm{N}^{\circ} 1$ y $\mathrm{N}^{\circ} 6$ alcanzaron profundidades entre 63,9 y 70,4 m; los anzuelos $\mathrm{N}^{\circ} 2$ y $\mathrm{N}^{\circ} 5$ entre 89,8 y $103,8 \mathrm{~m}$; en tanto que los anzuelos centrales $\mathrm{N}^{\circ} 3$ y $\mathrm{N}^{\circ} 4$ alcanzaron profundidades entre 103,2 y 133,1 m, para distintas velocidades de calado $\left(\mathrm{V}_{\mathrm{L}}\right)$ y proporción o tasa de recorte (k). Se observó que a medida que disminuyó la velocidad de calado aumentó la profundidad de trabajo de los anzuelos, es decir, existió una relación inversa con la tasa de recorte (Fig. 5).

Habiendo establecido que el peso total de una sección de palangre prototipo fue de $14,7 \mathrm{~kg}$, sobre la base de diez mediciones en el panel de simulación, se determinaron los valores promedio de tensión en la unión de la línea madre del prototipo en el apoyo (A), los cuales fluctuaron entre 2,42 kilogramos fuerza $(\mathrm{kgf})( \pm 0,46 \mathrm{DE})$ y $5,35( \pm 1,10 \mathrm{DE})$. Estos mostraron una relación directa con los diferentes valores de tasa de recorte (excursionamiento), que fluctuaron entre una media de 55,3\% (para una velocidad de 4,9 nudos) y $80,5 \%$ (para una velocidad de 7,1 nudos) (Fig. 6). De igual modo, la tensión mostró una relación directa con los valores promedio de ángulos de ataque, en los terminales de la línea madre del prototipo, los cuales variaron entre $10,4^{\circ}( \pm 0,97 \mathrm{DE})$ y $29,75^{\circ}( \pm 1,59 \mathrm{DE})$ (Fig. 7$)$.

\section{DISCUSIÓN}

Los resultados muestran que mediante uso del método de imitación mecánica en laboratorio, con ayuda de la infraestructura adecuada, el margen de error para determinar la profundidad de la catenaria, disminuye por las condiciones estáticas del modelaje. Asimismo, Nemoto (1975) obtuvo un resultado similar en la simulación de una sección de palangre de cinco reinales, basado en el método de imitación mecánica, utilizando papel milimetrado e hilo torsionado para estimar el seno de la catenaria. De igual manera, Eslava \& Gaertner (1990) utilizaron el mismo método y los mismos procedimientos empíricos para determinar la profundidad de un palangre regular de seis anzuelos.

Suzuki et al. (1977), para su estudio de campo, al no contar con datos sobre mediciones reales de profundidad de anzuelos, recurrieron al método teórico de cálculo de la profundidad utilizando la ecuación (1). Para ello, al no disponer de datos sobre los ángulos de ataque $(\varphi)$, ni de tasas de recorte $(\mathrm{k})$, tomaron como datos representativos los sugeridos por Hanamoto (1974) y Fuji \& Okamoto (1971): valores de tasas de recorte $\mathrm{k}$ igual a $60 \%$, para un ángulo de ataque aproximado $\varphi$ de $72^{\circ}$. En tal sentido, las profundidades de anzuelos máximas calcu- 

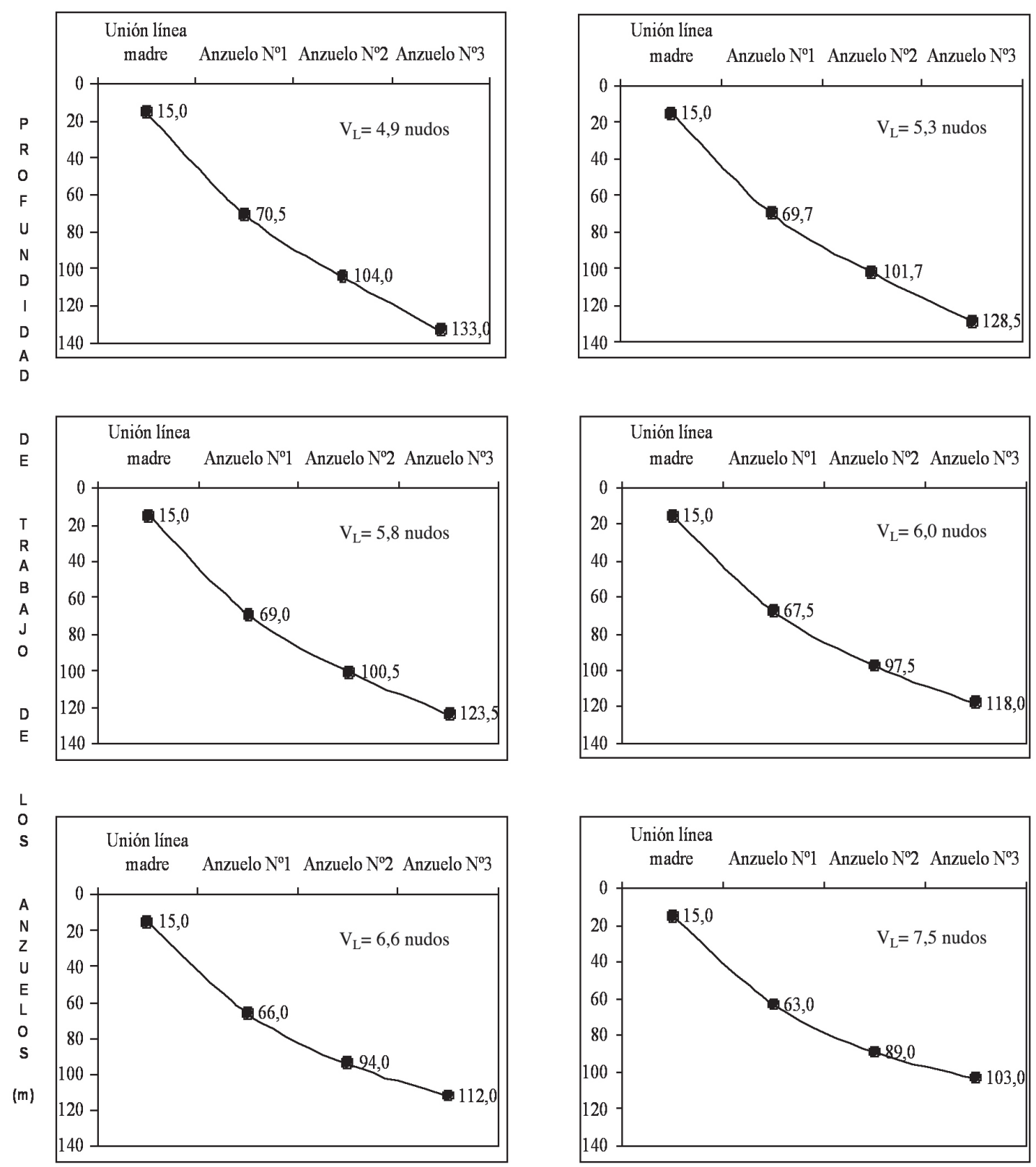

Figura 4. Profundidades de trabajo de los anzuelos con distintas velocidades de calado del palangre $\left(\mathrm{V}_{\mathrm{L}}\right)$. Figure 4. Operational depths of hooks for several speed at which the longline is set $\left(V_{L}\right)$. 


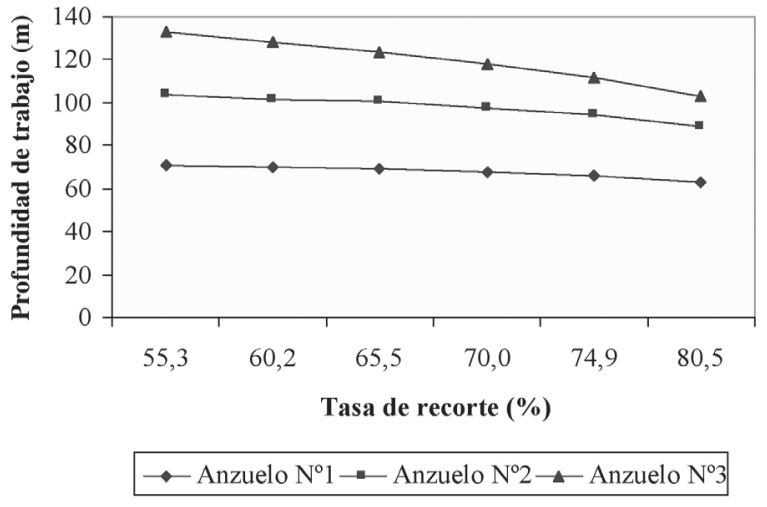

Figura 5. Relación entre la profundidad de trabajo de los anzuelos y la tasa de recorte del palangre.

Figure 5. Relationship between operational depth of hooks and the sagging rate of the longline.

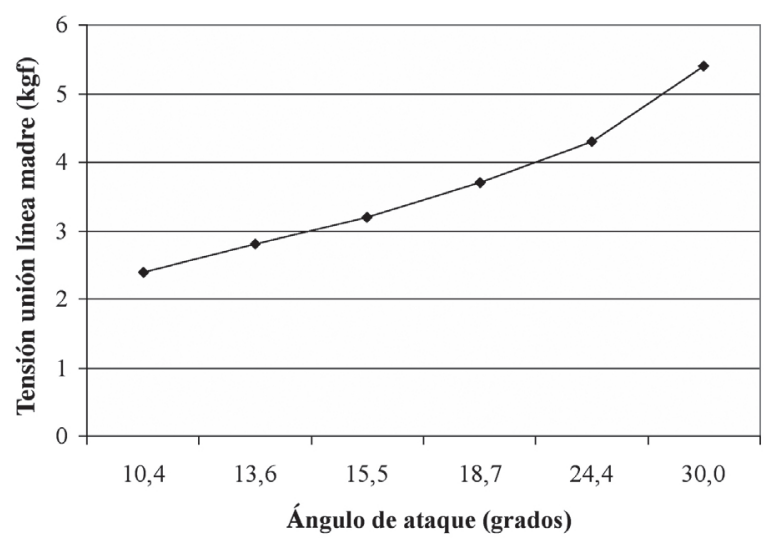

Figura 6. Relación entre el ángulo de ataque y tensión en la unión de la línea madre del palangre prototipo.

Figure 6. Relationship between the angle of incidence and the tension at the end of the main line of longline prototype.

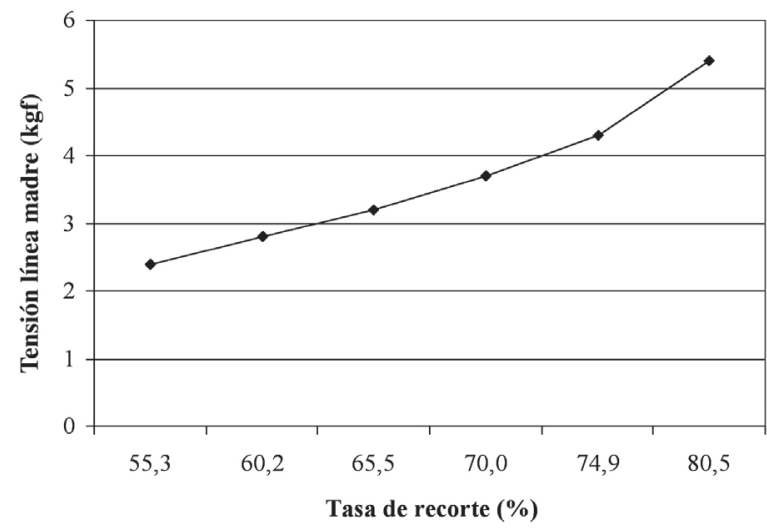

Figura 7. Relación entre la tasa de recorte y tensión en el terminal del prototipo del palangre.

Figure 7. Relationship between the sagging rate and tension at the end of the main line of longline prototype. ladas por el método teórico, con una tasa k igual a $60 \%$, estuvieron alrededor de $170 \mathrm{~m}$ para el palangre regular de 6 anzuelos, con valores constantes del cabo de baliza igual a $20 \mathrm{~m}$ y reinales igual a $30 \mathrm{~m}$, con una distancia entre boyas cercana a $50 \mathrm{~m}$. No obstante, las profundidades efectivas observadas por Hanamoto (1974), fueron consideradas menos profundas de las calculadas, por lo menos de 30 a $50 \mathrm{~m}$, debido principalmente a la fuerza de las corrientes marinas.

Las profundidades reales de captura han sido medidas en varios trabajos con ayuda de TDR (Time-Depth Recorder), entre los que se encuentra Yuwaki et al. (1985). Estos autores realizaron estudios de profundidad de anzuelos al sur de las Islas Hawaii, mediante el cálculo de la catenaria, utilizando la expresión de Yoshihara (1951) descrita en la ecuación (1). Estas profundidades se compararon con las profundidades reales obtenidas mediante un auto-registrador de profundidad (BS-04), para un palangre atunero de cinco anzuelos. Ellos encontraron que los valores reales de profundidad del anzuelo $\mathrm{N}^{\circ} 1$ y $\mathrm{N}^{\circ} 2$ son casi siempre similares a los valores de la catenaria calculada, pero los valores reales de profundidad encontrados respecto al anzuelo $\mathrm{N}^{\circ} 3$ son menores que los valores de la catenaria calculada. Esto indica que existe un margen de error significativo debido, entre otros aspectos, a la diferencia entre la profundidad de los anzuelos y la longitud de los reinales, así como por la diferencia de velocidad de la corriente del mar a distintas profundidades, como lo señala Hanamoto (1974).

Por otra parte, Nishi (1990) determinó las variaciones de profundidad por cada hora en un palangre atunero de cinco anzuelos, con ayuda de un registrador de profundidad. Estos resultados se compararon con las profundidades calculadas mediante el método teórico dado por Yoshihara (1951), que supone que la línea madre forma una catenaria. Las profundidades registradas en los anzuelos $\mathrm{N}^{\circ} 1$ y N $\mathrm{N}^{\circ}$ estuvieron entre 69 y $103 \mathrm{~m}$ (media de $89 \mathrm{~m}$ ), en los anzuelos $\mathrm{N}^{\circ} 2$ y N $\mathrm{N}^{\circ} 4$ entre 111 y $154 \mathrm{~m}$ (media de $134 \mathrm{~m}$ ) y en el anzuelo $\mathrm{N}^{\circ} 3$, entre 122 y $178 \mathrm{~m}$ (media de $154 \mathrm{~m}$ ). Los patrones de variación de los registros de profundidad por cada hora se clasificaron en tres categorías, $\mathrm{A}, \mathrm{B}$ y $\mathrm{C}$, donde estas dos últimas representaron las máximas velocidades de corriente obtenidas por medio de la deriva del palangre. Por lo tanto, las máximas diferencias entre las profundidades de anzuelos medidas y calculadas teó- 
ricamente, resultaron en promedio $16 \mathrm{~m}$ en los anzuelos $\mathrm{N}^{\mathrm{o}} 1$ y 5 , y $20 \mathrm{~m}$ en los anzuelos $\mathrm{N}^{\circ} 2,3$ y 4.

Asimismo, Misuno et al. (1997) estimando una serie de posibilidades de configuración de un palangre atunero sumergido, utilizando el método tridimensional, determinaron que la forma más aproximada a la línea madre en posición de trabajo, fue una curva menos pronunciada, la cual se obtuvo mediante la optimización de un algoritmo numérico. La validación de este método se logró mediante una serie de operaciones experimentales con el palangre, utilizando de 13 a 14 micro-BT por sección de línea madre, realizando observaciones oceanográficas permanentes, con ayuda de ADCP (Acoustic Doppler Current Profiler) y XBT (Expendable Bathythermograph). Las formas de línea madre obtenidas fueron consistentes con los patrones de las corrientes detectadas con ADCP.

También, Uozumi \& Okamoto (1997) monitorearon la profundidad de los palangres japoneses entre 5 y 15 anzuelos, utilizando TDR. Ellos encontraron que en una sección de 13 anzuelos, el más profundo de ellos resultó ser menor que la reportada por Suzuki et al. (1977). Además, observaron pequeñas diferencias en profundidad $(<30 \mathrm{~m})$ entre los anzuelos más profundos de las secciones en una misma calada, con las demás caladas alcanzando • 200 m, mostrando una gran variación. Estos autores notaron grandes diferencias (70-300 m) entre las profundidades de los anzuelos correspondientes, provenientes de diferentes caladas, debido a la variación en el tipo de embarcación utilizada, velocidad de lance, efecto del viento y la corriente, y distancia entre las boyas.

Al respecto, cabe señalar que Boggs (1992) observó mediante el uso de TDR, que las profundidades de los anzuelos generalmente eran menos profundas entre un $55 \%$ y un $68 \%$, que aquellas determinadas por medio de la catenaria. Asimismo, Hampton et al. (1998) determinaron la profundidad aproximada de distribución del palangre atunero para la pesca del atún ojo grande (Thunnus obesus) considerando el número de anzuelos entre flotadores, utilizando los análisis realizados por Suzuki et al. (1977), sobre la tasa de recorte de la línea madre y su influencia sobre la profundidad de un palangre regular de seis anzuelos. Estos autores también agregaron que, a la luz de los estudios reportados anteriormente, resulta dudoso que la profundidad de anzuelo pueda predecirse con certeza sin el uso del TDR.
Por otro lado, Santana-Hernández et al. (1998) aplicaron un método práctico para estimar las profundidades teóricas de operación de los anzuelos de un palangre tipo atunero. Ellos supusieron que la catenaria formada por la línea madre puede aproximarse, para fines prácticos, mediante la ecuación de la parábola con vértice en el origen $\left(\mathrm{y}=\mathrm{a} * \mathrm{x}^{2}\right)$. Este método estima sólo la profundidad ideal máxima de operación, sin considerar fuerzas diferentes a la de gravedad, como son el efecto de las corrientes marinas y las tensiones provocadas por los organismos capturados, las cuales provocan una deformación de la parábola descrita por la línea madre. Estas estimaciones teóricas fueron validadas con ayuda de un CTD instalado en los anzuelos, que además de registrar la profundidad, registraba la temperatura y salinidad con intervalos de 10 minutos, durante 8 horas. Los resultados indican que las profundidades calculadas resultan mayores que el promedio de las profundidades medidas con un CTD para cada anzuelo. En el mismo sentido, Bigelow et al. (2000) para predecir la profundidad de captura del palangre, consideraron todos los elementos que influyen sobre la configuración de la línea madre, tales como la longitud de la línea de flotación, longitud de los cabos de balizas, distancia entre éstas, tasa de recorte de la línea madre y una variedad de factores ambientales, particularmente el viento y las corrientes.

Aunque las profundidades de captura reales han sido determinadas en diferentes estudios con ayuda de los TDR, los datos sobre mediciones de profundidad de captura raramente se encuentran disponibles en las estadísticas de caladas de los palangreros comerciales. Sin embargo, el número de anzuelos entre flotadores, son registros rutinarios en las embarcaciones japonesas, los cuales pueden ser utilizados, junto con información sobre configuración de calada, para estimar la profundidad aproximada de los anzuelos, mediante el modelo teórico de la catenaria utilizado por Suzuki et al. (1977).

En el laboratorio, mediante el método de imitación mecánica utilizado en la presente investigación, los valores promedio obtenidos en los anzuelos centrales ( $\mathrm{N}^{\circ} 3$ y $\left.\mathrm{N}^{\circ} 4\right)$, con una tasa de recorte $\mathrm{k}$ igual a $60,21 \%$, alcanzaron profundidades de $128,5 \mathrm{~m}$, con una constante de $h_{b}+h_{a}=33,25 \mathrm{~m}$. Estos resultados pueden ser comparados con los de Suzuki et al. (1977), para el palangre regular de seis anzuelos, dado que son próximos a los obtenidos para los anzuelos $\mathrm{N}^{\circ} 3$ y N 4 para un k igual a $60 \%$, si se consi- 
dera que la diferencia encontrada, entre $170 \mathrm{~m}$ (Suzuki et al., 1977) y 128,5 m (investigación actual), pudiera radicar en la longitud de los reinales $(30 \mathrm{~m})$, longitud de los cabos de boya $(20 \mathrm{~m}) \mathrm{y}$ distancia entre reinales $(50 \mathrm{~m})$. En el palangre estándar utilizado por la flota de Isla de Margarita, estas longitudes son iguales a 19,25 m, 14 m y 39 m, respectivamente.

Por consiguiente, si se comparan los valores de profundidad de anzuelos obtenidos por el método de imitación mecánica, con los resultados obtenidos por los autores antes citados, existe coincidencia en el sentido que, desde el punto de vista teórico, no se contemplan otros elementos como el efecto corriente y las tensiones ejercidas por los organismos capturados, que pueden causar una sobreestimación de las profundidades reales, alcanzando valores entre un $15 \%$ y $20 \%$ según lo señalado por Suzuki et al. (1977), Yuwaki (1985), Nishi (1990) y Santana-Hernández (1998). Sin embargo, mediante el uso del método de simulación análoga, en el laboratorio también pueden ser considerados los parámetros de corriente y esfuerzo promedio ejercido por el pez, de acuerdo a lo sugerido por Fridman (1973), para obtener una mayor aproximación a las profundidades reales. Además, igualmente es necesario estudiar el diseño del arte de pesca para su perfeccionamiento e investigar las condiciones oceanográficas del área de trabajo.

\section{AGRADECIMIENTOS}

El presente trabajo se realizó gracias al financiamiento parcial del Consejo de Investigación de la Universidad de Oriente, Núcleo Nueva Esparta, al Proyecto CI-4-1703-0621/99, "Identificación de los puntos críticos de operación a bordo de una embarcación de altura tipo palangrera atunera de la Isla de Margarita”. Un reconocimiento muy especial al Sr. Darío González, por permitir abordar su lancha "Don Chicho II" utilizada como embarcación prototipo para el desarrollo de los objetivos del proyecto; así como al patrón de la embarcación, Sr. Hilario González, por la valiosa colaboración prestada durante el desarrollo de las actividades a bordo, junto a su tripulación. A Luis León y Luis Troccoli por la lectura crítica del manuscrito y sus recomendaciones y a José Alió, por la lectura crítica y revisión de la traducción al inglés del manuscrito.

\section{REFERENCIAS}

Baranov, F.I. 1939. Primienieniye metoda mejanícheskoy imitatsii pri raschiotax ribolovnix orudii. Ed. Trudy Mosrybtuza, Vuip., 2: 103-130.

Baranov, F.I. 1969. Selected works on fishings gear. Vol. 1. Comercial Fishing Techniques. Israel Program for Scientific Translations, Jerusalem, 631 pp.

Bigelow, K., J. Hampton \& N. Miyabe. 2000. Application of a habitat-based model to estimate effective longline fishing effort and relative abundance of Pacific bigeye tuna (Thunnus obesus). Working Paper BET-1, $13^{\text {th }}$ Meeting of the Standing Committee on Tuna and Billfish. SCTB 13, Noumea, New Caledonia, 18 pp.

Boggs, C.H. 1992. Depth, capture time, and hooked longevity of longline-caught pelagic fish: Timing bites of fish with chips. U.S. Fish. Bull., 90: 642658 .

Eslava, N. \& D. Gaertner, 1990. Distribución vertical de los atunes y especies de pico y su abundancia en el Mar Caribe. ICCAT, Coll. Vol. Sci. Pap., 32(1): 39-47.

Fridman, A.L. 1973. Theory and design of commercial fishing gear. Israel Program for Scientific Translations, Jerusalem, 489 pp.

Fridman, A.L. 1986. Calculation for fishing gear designs. FAO Fishing Manuals. Fishing News Books Ltd., Farnham Surrey, 241 pp.

Fuji, I. \& H. Okamoto. 1971. On the shapes of tuna longline gear under water and catches of tunas billfishes. Showa 44 nendo Mie-ken Hamajima Suishi Jigyo Hokoku, pp. 45-103 (En japonés con resumen en inglés).

Hampton, J., K. Bigelow \& M. Labelle. 1998. Effect of longline fishing depth, water temperature and dissolved oxygen on bigeye tuna (Thunnus obesus) abundance indices. Oceanic Fisheries Programme Secretariat of the Pacific Community. B.P. D5, Noumea, New Caledonia, 17 pp. (working paper).

Hanamoto, E. 1974. Fishery oceanography of bigeye tuna - I. Depth of capture by tuna longline gear. En: The Eastern Tropical Pacific Ocean. La Mer (Bulletin de la Société Franco-Japonaise d'Oceanographie), 12(3): 10-18 (En japonés con resumen en inglés). 
Martínez, G. \& T. Melo. 1990. Dimensionamiento de aparejos de pesca mediante simulación mecánica. Estud. Doc., Univ. Católica Valparaíso, 17/ 90: $68 \mathrm{pp}$.

Martínez, G. \& L. Carroza. 1992. Configuración del cabo de fondeo de un long-line de cultivo a través de simulación análoga. Invest. Mar., Valparaíso, 20: 55-66.

Martínez, G., T. Melo \& L. Rodríguez. 1993. Análisis de las metodologías de dimensionamiento de artes y aparejos de pesca utilizados en Chile. Estud. Doc., Univ. Católica Valparaíso, 8/93: 127 pp.

Matsumoto, T., Y. Uozumi, K. Uosaki \& M. Okazaki. 1999. Preliminary review of billfish hooking depth measured by small bathythermograph systems attached to longline gear. Col. Vol. Sci. Pap. ICCAT, 53: 337-344.

Misuno, K., M. Okazaki, H. Nakano \& H. Okamura. 1997. Estimation of underwater shape of tuna longline by using micro-BTs. Bull. Nat. Res. Inst. Far. Seas., 34: 1-25 (En japonés con resumen en inglés).

Nakano, H., M. Okazaki \& H. Okamoto. 1997. Analysis of catch depth by species for tuna longline fishery based on catch by branch lines. Bull. Nat. Res. Inst. Far Seas Fish., 34: 43-62.

Nemoto, T. 1975. Pesca de atún por palangre. Inf. Tec. $\mathrm{N}^{\circ}$ 1. Ministerio de Agricultura y Cría. Oficina Nacional de Pesca, Caracas, 30 pp.

Nishi, T. 1990. The hourly variations of the depth of hooks and hooking depth of yellowfin tuna (Thunnus albacares), and bigeye tuna (Thunnus obesus), of tuna longline in the eastern region of the Indian Ocean. Mem. Fac. Fish. Kagoshima Univ., 39: 81-98 (En japonés con resumen en inglés).
Okazaki, M., K. Mizuno, T. Watanabe \& Sh. Yanagi. 1997. Improved model of micro bathythermograph system for tuna longline boats and its application to fisheries oceanography. Bull. Nat. Res. Inst. Far Seas Fish., 34: 25-42.

Santana-Hernández, H., R. Macías-Zamora \& J.J. Valdez-Flores. 1998. Selectividad del sistema de palangre utilizado por la flota mexicana en la Zona Económica Exclusiva. Ciencias Marinas, 24(2): 193-210.

Suzuki, Z., Y. Warashina \& M. Kishida. 1977. The comparison of catches by regular and deep tuna longline gears in Western and Central Equatorial Pacific. Bull. Far. Seas. Fish. Res. Lab., 15: 51-89.

Uozumi, Y. \& H. Okamoto. 1997. Research on the hook depth of longline gear in the 1995 research cruise of the R/V Shoyo Maru. Working paper 3, $7^{\text {th }}$ Meeting of the Western Pacific Yellowfin Research Group, Nadi, Fiji, 20 pp.

Voinikanis-Mirskii, V.N. 1979. Teoría y diseño de artes de la pesca industrial. Material Didáctico. Instituto Técnico de la Industria y Economía Pesquera de Astrakhan. Ministerio de Pesquería URSS. Cátedra de Pesca Industrial, 14: 1-14 (En ruso).

Yokawa, K. \& Y. Takuchi. 2000. Estimation of abundance index of the white marlin caught by japanese longliners in the Atlantic Ocean. ICCAT Scrs. Doc. Scrs/02/060, 21 pp.

Yoshihara, T. 1951. Distribution of fishes caught by the longline. II. Vertical distribution. Bull. Jap. Soc. Sci. Fish., 16(8): 370-374 (En japonés).

Yuwaki, Y., M. Higashi, K. Shimada \& T. Henmi. 1985. Studies on the depth of longline hook. Mem. Fac. Fish. Kagoshima Univ., 34(1): 21-26. 